PROCEEDINGS OF THE

AMERICAN MATHEMATICAL SOCIETY

Volume 140, Number 12, December 2012, Pages 4217-4225

S 0002-9939(2012)11280-8

Article electronically published on April 12, 2012

\title{
BLOCH-TO-BMOA COMPOSITIONS ON COMPLEX BALLS
}

\author{
EVGUENI DOUBTSOV
}

(Communicated by Richard Rochberg)

\begin{abstract}
Let $\varphi$ be a holomorphic map between complex unit balls. We characterize those $\varphi$ for which the composition operator $f \mapsto f \circ \varphi$ maps the Bloch space into BMOA.
\end{abstract}

\section{INTRODUCTION}

Let $H\left(B_{m}\right)$ denote the space of holomorphic functions in the unit ball $B_{m}$ of $\mathbb{C}^{m}, m \geq 1$.

1.1. Spaces under consideration. The Bloch space $\mathcal{B}\left(B_{m}\right)$ consists of those functions $f \in H\left(B_{m}\right)$ for which

$$
\|f\|_{\mathcal{B}\left(B_{m}\right)}=|f(0)|+\sup _{w \in B_{m}}|\mathcal{R} f(w)|\left(1-|w|^{2}\right)<\infty
$$

where

$$
\mathcal{R} f(w)=\sum_{j=1}^{m} w_{j} \frac{\partial f}{\partial w_{j}}(w), \quad w \in B_{m},
$$

is the radial derivative of $f$. The Hardy space $H^{p}\left(B_{n}\right), p>0, n \geq 1$, consists of functions $f \in H\left(B_{n}\right)$ such that

$$
\|f\|_{H^{p}\left(B_{n}\right)}^{p}=\sup _{0<r<1} \int_{\partial B_{n}}|f(r \zeta)|^{p} d \sigma_{n}(\zeta)<\infty,
$$

where $\sigma_{n}$ is the normalized Lebesgue measure on the sphere $\partial B_{n}$. Also, we consider $\operatorname{BMOA}\left(B_{n}\right)$, the space of holomorphic functions that have bounded mean oscillation on $\partial B_{n}$. Equivalent definitions of $\operatorname{BMOA}\left(B_{n}\right)$ are given in Section 2 ,

1.2. Composition operators. Given a holomorphic map $\varphi: B_{n} \rightarrow B_{m}$, the composition operator $C_{\varphi}: H\left(B_{m}\right) \rightarrow H\left(B_{n}\right)$ is defined by the following identity:

$$
\left(C_{\varphi} f\right)(z)=f(\varphi(z)), \quad f \in H\left(B_{m}\right), z \in B_{n} .
$$

Various properties of $C_{\varphi}$ are presented in the monographs [5, 12]. In this paper, we describe those $\varphi$ for which $C_{\varphi} \operatorname{maps} \mathcal{B}\left(B_{m}\right)$ into $\operatorname{BMOA}\left(B_{n}\right)$.

Received by the editors January 28, 2011 and, in revised form, May 25, 2011.

2010 Mathematics Subject Classification. Primary 32A18, 32A37; Secondary 32H02, 47B33.

Key words and phrases. Bloch space, BMOA, composition operator.

This research was supported by RFBR (grant No. 11-01-00526).

(C)2012 American Mathematical Society Reverts to public domain 28 years from publication 
1.2.1. Bloch-to-BMOA composition operators: $m=1$. There is a series of results about the operators under consideration. In particular, characterizations of the bounded operators $C_{\varphi}: \mathcal{B}\left(B_{1}\right) \rightarrow \operatorname{BMOA}\left(B_{n}\right)$ were obtained in [10]; see also [7, 9], 13. The cases $n=1$ and $n \geq 2$ are rather different. Indeed, let $\varphi: B_{n} \rightarrow B_{1}$ be a holomorphic Lipschitz function of order 1 . Then $C_{\varphi}$ does not map $\mathcal{B}\left(B_{1}\right)$ into $\operatorname{BMOA}\left(B_{n}\right)$ when $\|\varphi\|_{\infty}=1$ and $n=1$, but $C_{\varphi}$ maps $\mathcal{B}\left(B_{1}\right)$ into $\operatorname{BMOA}\left(B_{n}\right)$ when $n \geq 2$ (see [4] and [10], respectively). Motivations and further references are given in $[3,10$.

1.2.2. Bloch-to-BMOA composition operators: $m \geq 2$. To the best knowledge of the author, for arbitrary $n, m \in \mathbb{N}$, the problem in question was considered only in [3], where the bounded and compact composition operators $C_{\varphi}: \mathcal{B}\left(B_{m}\right) \rightarrow \operatorname{BMOA}\left(B_{n}\right)$ are characterized under an additional regularity assumption about $\varphi$. Namely, the operator $C_{\varphi}$ is bounded if and only if

$$
\frac{\left(1-|z|^{2}\right)|\mathcal{R} \varphi(z)|^{2}}{\left(1-|\varphi(z)|^{2}\right)^{2}} d \nu_{n}(z) \quad \text { is a Carleson measure, }
$$

where $\nu_{n}$ is Lebesgue measure on $B_{n}$ and $\nu_{n}\left(B_{n}\right)=1$.

In the present paper, we use the Möbius-invariance of the spaces $\operatorname{BMOA}\left(B_{n}\right)$ and $\mathcal{B}\left(B_{m}\right)$. So, for $z \in B_{n}$, let $\phi_{z}$ denote the involution of $B_{n}$ such that $\phi_{z}(0)=z$. Let $\beta_{m}$ denote the Bergman metric on the ball $B_{m}$. The main result of this paper is the following theorem:

Theorem 1.1. Let $\varphi: B_{n} \rightarrow B_{m}$ be a holomorphic map. Then the following properties are equivalent:

$$
\begin{gathered}
C_{\varphi}: \mathcal{B}\left(B_{m}\right) \rightarrow \operatorname{BMOA}\left(B_{n}\right) \text { is a bounded operator; } \\
\sup _{z \in B_{n}} \sup _{0<r<1} \int_{\partial B_{n}} \beta_{m}\left(\varphi\left(\phi_{z}(r \zeta)\right), \varphi(z)\right) d \sigma_{n}(\zeta)<\infty .
\end{gathered}
$$

For $m=1$, Theorem 1.1 was proved in [7]; see also [10].

\subsection{Comments.}

1.3.1. Property (1.3) and hyperbolic BMOA. Recall that the Garsia seminorm on $\operatorname{BMOA}\left(B_{n}\right)$ is defined by the identity

$$
\|f\|_{G^{1}\left(B_{n}\right)}=\sup _{z \in B_{n}} \sup _{0<r<1} \int_{\partial B_{n}}\left|f\left(\phi_{z}(r \zeta)\right)-f(z)\right| d \sigma_{n}(\zeta) .
$$

Therefore, (1.3) reduces to the property $\|f\|_{G^{1}\left(B_{n}\right)}<\infty$ when $\varphi$ is replaced by $f \in H\left(B_{n}\right)$ and $\beta_{m}$ is replaced by the Euclidean metric. So, as in [9, 14] for $n=m=1$, we say that (1.3) defines the hyperbolic BMOA class. However, other names have been used for this class; see [7].

1.3.2. Carleson measures. As observed in [3], the implication $(1.1) \Rightarrow(1.2)$ holds for all holomorphic maps $\varphi: B_{n} \rightarrow B_{m}$. Hence, Theorem 1.1 guarantees that (1.1) implies (1.3) for arbitrary $\varphi$. So, one could expect that (1.3) implies (1.1) for all $\varphi$. If this is the case, then it would be interesting to find a direct proof of the implication in question. 
1.3.3. Bounded mean oscillation. The classical seminorm on $\operatorname{BMOA}\left(B_{n}\right)$ is defined by the identity

$$
\|f\|_{\mathrm{BMOA}\left(B_{n}\right)}=\sup \frac{1}{\sigma_{n}(Q)} \int_{Q}\left|f^{*}-f_{Q}^{*}\right| d \sigma_{n},
$$

where $f^{*}$ is the boundary function of $f, f_{Q}^{*}$ is the average of $f^{*}$ over $Q$, and the supremum is taken over all quasi-balls $Q=Q_{r}(\eta)=\left\{\xi \in \partial B_{n}:|1-\langle\eta, \xi\rangle|<r\right\}$, $\eta \in \partial B_{n}$; see Section 2.4 for details.

The hyperbolic analog of the property $\|f\|_{\mathrm{BMOA}\left(B_{n}\right)}<\infty$ is the following one:

$$
\sup _{r>0, \eta \in \partial B_{n}} \frac{1}{Q_{r}(\eta)} \int_{Q_{r}(\eta)} \beta_{m}\left(\varphi^{*}(\zeta), \varphi\left(\eta \sqrt{1-r^{2}}\right)\right) d \sigma_{n}(\zeta)<\infty .
$$

The relations between (1.3), (1.4) and similar properties will be considered elsewhere.

\section{Preliminaries}

Basic properties of $\mathcal{B}\left(B_{m}\right)$ and $\mathrm{BMOA}\left(B_{n}\right)$ are collected in this section. Further details are given in [1, 15]; see also [6] for $n=m=1$.

2.1. Automorphisms of the unit ball. The automorphism group of $B_{n}$, denoted by $\operatorname{Aut}\left(B_{n}\right)$, consists of all biholomorphic mappings from $B_{n}$ onto $B_{n}$. Given $z \in B_{n}$, the involution (or the Möbius transform) $\phi_{z} \in \operatorname{Aut}\left(B_{n}\right)$ is defined for $\lambda \in B_{n}$ as follows:

$$
\begin{aligned}
& \phi_{z}(\lambda)=-\lambda \quad \text { when } z=0, \\
& \phi_{z}(\lambda)=\frac{z-P_{z} \lambda-\sqrt{1-|z|^{2}} Q_{z} \lambda}{1-\langle\lambda, z\rangle} \quad \text { when } z \in B_{n} \backslash\{0\},
\end{aligned}
$$

where $P_{z} \lambda=|z|^{-2}\langle\lambda, z\rangle z, Q_{z} \lambda=\lambda-P_{z} \lambda$. To distinguish the involutions of $B_{m}$, we write $\psi_{w}, w \in B_{m}$ in the place of $\phi_{z}, z \in B_{n}$.

2.2. Hyperbolic BMOA. The hyperbolic BMOA is defined by (1.3) in terms of the Bergman metric $\beta_{m}$ on $B_{m}$. Note that

$$
\beta_{m}\left(w_{1}, w_{2}\right)=C \log \frac{1+\left|\psi_{w_{1}}\left(w_{2}\right)\right|}{1-\left|\psi_{w_{1}}\left(w_{2}\right)\right|}, \quad w_{1}, w_{2} \in B_{m} .
$$

So, a holomorphic map $\varphi: B_{n} \rightarrow B_{m}$ is in the hyperbolic BMOA if and only if

$$
\sup _{z \in B_{n}} \sup _{0<r<1} \int_{\partial B_{n}} \log \frac{1}{1-\left|\psi_{\varphi(z)}\left(\varphi\left(\phi_{z}(r \zeta)\right)\right)\right|^{2}} d \sigma_{n}(\zeta)<\infty .
$$

2.3. Seminorms on the Bloch space. For $f \in H\left(B_{m}\right)$, put

$$
|\widetilde{\nabla} f(w)|^{2}=\left(1-|w|^{2}\right)\left(|\nabla f(w)|^{2}-|\mathcal{R} f(w)|^{2}\right), \quad w \in B_{m},
$$

where

$$
\nabla f(w)=\left(\frac{\partial f}{\partial w_{1}}(w), \ldots, \frac{\partial f}{\partial w_{m}}(w)\right)
$$

is the complex gradient of $f$. 
Let $\widetilde{\mathcal{B}}\left(B_{m}\right)$ denote the quotient of $\mathcal{B}\left(B_{m}\right)$ by the space of constant functions. Then $\widetilde{\mathcal{B}}\left(B_{m}\right)$ is a Banach space with respect to the following norms:

$$
\begin{aligned}
& \sup _{w \in B_{m}}|\mathcal{R} f(w)|\left(1-|w|^{2}\right) ; \\
& \sup _{w \in B_{m}}|\nabla f(w)|\left(1-|w|^{2}\right) ; \\
& \|f\|_{\widetilde{\mathcal{B}}\left(B_{m}\right)}=\sup _{w \in B_{m}}|\widetilde{\nabla} f(w)| .
\end{aligned}
$$

Clearly, the above expressions are seminorms on $\mathcal{B}\left(B_{m}\right)$; these seminorms degenerate exactly on the constant functions. The main advantage of $\|\cdot\|_{\widetilde{\mathcal{B}}\left(B_{m}\right)}$ is its Möbius-invariance. Namely,

$$
\|f \circ \psi\|_{\widetilde{\mathcal{B}}\left(B_{m}\right)}=\|f\|_{\widetilde{\mathcal{B}}\left(B_{m}\right)}
$$

for all $\psi \in \operatorname{Aut}\left(B_{m}\right), f \in \mathcal{B}\left(B_{m}\right)$

Also, a function $f \in H\left(B_{m}\right)$ belongs to $\mathcal{B}\left(B_{m}\right)$ if and only if there exists a constant $C>0$ such that

$$
\left|f\left(w_{1}\right)-f\left(w_{2}\right)\right| \leq C \beta_{m}\left(w_{1}, w_{2}\right) \quad \text { for all } w_{1}, w_{2} \in B_{m} .
$$

2.4. Seminorms on BMOA. For $\zeta \in \partial B_{n}$ and $r>0$, put

$$
Q_{r}(\zeta)=\left\{\xi \in \partial B_{n}:|1-\langle\zeta, \xi\rangle|<r\right\} .
$$

Recall that the radial limits $\left|f^{*}(\zeta)\right|=\lim _{r \rightarrow 1-}|f(r \zeta)|$ are defined for $\sigma_{n}$-almost all $\zeta \in \partial B_{n}$ for every $f \in H^{1}\left(B_{n}\right)$. Let $\operatorname{BMOA}\left(B_{n}\right)$ denote the space of functions $f \in H^{1}\left(B_{n}\right)$ such that

$$
|f(0)|^{p}+\sup _{\zeta \in \partial B_{n}, r>0} \frac{1}{\sigma_{n}(Q)} \int_{Q}\left|f^{*}-f_{Q}^{*}\right|^{p} d \sigma_{n}<\infty,
$$

where $p=1, Q=Q_{r}(\zeta)$ and

$$
f_{Q}^{*}=\frac{1}{\sigma_{n}(Q)} \int_{Q} f^{*} d \sigma_{n} .
$$

The norm $\|f\|_{\mathrm{BMOA}\left(B_{n}\right)}$ is defined as the left-hand side of (2.3) with $p=1$.

By the John-Nirenberg theorem, there exist constants $A(n)>0$ and $C(n)>0$ such that

$$
\int_{\partial B_{n}} \exp \left(\left|f^{*}(\zeta)\right|\right) d \sigma_{n}(\zeta) \leq C(n)
$$

for all $f \in \operatorname{BMOA}\left(B_{n}\right)$ with $\|f\|_{\mathrm{BMOA}\left(B_{n}\right)} \leq A(n)$. The John-Nirenberg inequality guarantees that $f \in \operatorname{BMOA}\left(B_{n}\right)$ if and only if $f \in H^{2}\left(B_{n}\right)$ and (2.3) holds with $p=2$.

The proof of Theorem 1.1 will be based on the following fact: (2.3) holds for $p=1$ or for $p=2$ if and only if

$$
\|f\|_{G^{p}\left(B_{n}\right)}=\sup _{z \in B_{n}}\left\|f \circ \phi_{z}-f(z)\right\|_{H^{p}\left(B_{n}\right)}<\infty
$$

for $p=1$ or for $p=2$. The above seminorms degenerate exactly on the constant functions. Let $\widehat{\operatorname{BMOA}}\left(B_{n}\right)$ denote the quotient of $\operatorname{BMOA}\left(B_{n}\right)$ by the space of constant functions. Then $\widetilde{\mathrm{BMOA}}\left(B_{n}\right)$ is a Banach space with respect to the Garsia norm $\|\cdot\|_{G^{p}\left(B_{n}\right)}, p=1$ or $p=2$. 


\section{Auxiliary REsults}

3.1. Aleksandrov-Ryll-Wojtaszczyk polynomials. Ryll and Wojtaszczyk [1] constructed holomorphic polynomials which proved to be very useful for many problems of function theory in the unit ball. We need the following improvement of the Ryll-Wojtaszczyk theorem.

Theorem 3.1 ([2, Theorem 4]). Let $m \in \mathbb{N}$. Then there exists $\delta=\delta(m) \in(0,1)$ and $J=J(m) \in \mathbb{N}$ with the following property: For every $d \in \mathbb{N}$, there exist holomorphic homogeneous polynomials $W_{j}[d]$ of degree $d, 1 \leq j \leq J$, such that

$$
\begin{aligned}
\left\|W_{j}[d]\right\|_{L^{\infty}\left(\partial B_{m}\right)} \leq 1 & \text { and } \\
\max _{1 \leq j \leq J}\left|W_{j}[d](\xi)\right| \geq \delta & \text { for all } \xi \in \partial B_{m} .
\end{aligned}
$$

3.2. A reverse estimate for Bloch functions. For $m=1$, it is known that the following lemma holds with $J(1)=1$; see [8, Lemma 2.1].

Lemma 3.2. Let $m \in \mathbb{N}$ and let $0<p<\infty$. Then there exist constants $J=$ $J(m) \in \mathbb{N}, \tau_{m, p}>0$ and there exist functions $F_{j, x} \in \mathcal{B}\left(B_{m}\right), 1 \leq j \leq J, 0 \leq x \leq 1$, such that $\left\|F_{j, x}\right\|_{\mathcal{B}\left(B_{m}\right)} \leq 1, F_{j, x}(0)=0$, and

$$
\sum_{j=1}^{J} \int_{0}^{1}\left|F_{j, x}(w)\right|^{p} d x \geq \tau_{m, p}\left(\log \frac{1}{1-|w|^{2}}\right)^{\frac{p}{2}}
$$

for all $w \in B_{m}$.

Proof. Let the constant $\delta \in(0,1)$ and the polynomials $W_{j}[d], 1 \leq j \leq J, d \in \mathbb{N}$, be those provided by Theorem 3.1. For $k \in \mathbb{Z}_{+}$, let $R_{k}$ denote the Rademacher function:

$$
R_{k}(x)=\operatorname{sign} \sin \left(2^{k+1} \pi x\right), \quad x \in[0,1] .
$$

For each non-diadic $x \in[0,1]$, consider the functions

$$
F_{j, x}(w)=\frac{1}{4} \sum_{k=0}^{\infty} R_{k}(x) W_{j}\left[2^{k}\right](w), \quad w \in B_{m}, 1 \leq j \leq J .
$$

Estimate (3.1) guarantees that

$$
\left(1-|w|^{2}\right)\left|\left(\mathcal{R} F_{j, x}\right)(w)\right| \leq \frac{1-|w|^{2}}{4} \sum_{k=0}^{\infty} 2^{k}|w|^{2^{k}} \leq \frac{1-|w|^{2}}{2} \sum_{n=1}^{\infty}|w|^{n} \leq 1
$$

for all $w \in B_{m}$. Observe that $F_{j, x}(0)=0$; hence, $\left\|F_{j, x}\right\|_{\mathcal{B}\left(B_{m}\right)} \leq 1$.

Next,

$$
C_{p} \int_{0}^{1}\left|F_{j, x}(w)\right|^{p} d x \geq\left(\sum_{k=0}^{\infty}\left|W_{j}\left[2^{k}\right](w)\right|^{2}\right)^{\frac{p}{2}}
$$


by [16. Chapter V, Theorem 8.4]. Given positive numbers $a_{j}, 1 \leq j \leq J=J(m)$, we have

$$
\left(\sum_{j=1}^{J} a_{j}\right)^{\frac{p}{2}} \leq C_{p, m} \sum_{j=1}^{J} a_{j}^{p / 2}
$$

Hence,

$$
C_{p, m} \sum_{j=1}^{J} \int_{0}^{1}\left|F_{j, x}(w)\right|^{p} d x \geq\left(\sum_{k=0}^{\infty} \sum_{j=1}^{J}\left|W_{j}\left[2^{k}\right](w)\right|^{2}\right)^{\frac{p}{2}} .
$$

Recall that $W_{j}\left[2^{k}\right]$ is a homogeneous polynomial of degree $2^{k}$; thus,

$$
\begin{aligned}
\sum_{k=0}^{\infty} \sum_{j=1}^{J}\left|W_{j}\left[2^{k}\right](w)\right|^{2} & \geq \delta^{2} \sum_{k=0}^{\infty}|w|^{2^{k+1}} \\
& \geq \delta^{2} \sum_{n=1}^{\infty} \frac{|w|^{2 n}}{n}=\delta^{2} \log \frac{1}{1-|w|^{2}}, \quad w \in B_{m}
\end{aligned}
$$

by (3.2). So,

$$
\sum_{j=1}^{J} \int_{0}^{1}\left|F_{j, x}(w)\right|^{p} d x \geq\left(\frac{\delta^{2}}{C_{m, p}} \log \frac{1}{1-|w|^{2}}\right)^{\frac{p}{2}}
$$

as required.

\section{BLOCH-TO-BMOA COMPOSITION OPERATORS}

4.1. Proof of Theorem 1.1. Assume that (1.2) holds. Note that $C_{\varphi} 1=1$; hence, $C_{\varphi}: \widetilde{\mathcal{B}}\left(B_{m}\right) \rightarrow \widehat{\mathrm{BMOA}}\left(B_{n}\right)$ is a bounded operator. Using (2.5) with $p=2$, we obtain

$$
\sup _{z \in B_{n}} \sup _{0<r<1} \int_{\partial B_{n}}\left|f \circ \varphi \circ \phi_{z}(r \zeta)-f \circ \varphi(z)\right|^{2} d \sigma_{n}(\zeta) \leq C\|f\|_{\widetilde{\mathcal{B}}\left(B_{m}\right)}^{2} .
$$

Let the constant $\tau=\tau_{m, 2}>0$ and the functions $F_{j, x}, 1 \leq j \leq J, 0 \leq x \leq 1$, be those provided by Lemma 3.2 for $p=2$. Note that $\left\|F_{j, x}\right\|_{\widetilde{\mathcal{B}}\left(B_{m}\right)} \leq C\left\|F_{j, x}\right\|_{\mathcal{B}\left(B_{m}\right)} \leq C$. Recall that $\|\cdot\|_{\widetilde{\mathcal{B}}\left(B_{m}\right)}$ is Möbius-invariant; hence, $\left\|F_{j, x} \circ \psi_{\varphi(z)}\right\|_{\widetilde{\mathcal{B}}\left(B_{m}\right)} \leq C$, where the constant $C>0$ does not depend on $z \in B_{n}$. Also, we have $F_{j, x} \circ \psi_{\varphi(z)}(\varphi(z))=$ $F_{j, x}(0)=0$. Thus, by (4.1) with $f=F_{j, x} \circ \psi_{\varphi(z)}$,

$$
\int_{\partial B_{n}}\left|F_{j, x} \circ \psi_{\varphi(z)}\left(\varphi\left(\phi_{z}(r \zeta)\right)\right)\right|^{2} d \sigma_{n}(\zeta) \leq C
$$

for all $z \in B_{n}, 0<r<1,0 \leq x \leq 1$. Hence,

$$
\sum_{j=1}^{J} \int_{0}^{1} \int_{\partial B_{n}}\left|F_{j, x} \circ \psi_{\varphi(z)}\left(\varphi\left(\phi_{z}(r \zeta)\right)\right)\right|^{2} d \sigma_{n}(\zeta) d x \leq C, \quad z \in B_{n}, 0<r<1 .
$$

Therefore, Fubini's theorem and Lemma 3.2 guarantee that

$$
\tau \int_{\partial B_{n}} \log \frac{1}{1-\left|\psi_{\varphi(z)}\left(\varphi\left(\phi_{z}(r \zeta)\right)\right)\right|^{2}} d \sigma_{n}(\zeta) \leq C, \quad z \in B_{n}, 0<r<1
$$

So, we obtain (2.1) or, equivalently, (1.3). 
To prove the converse implication, assume that (1.3) holds, that is,

$$
\sup _{z \in B_{n}} \sup _{0<r<1} \int_{\partial B_{n}} \beta_{m}\left(\varphi\left(\phi_{z}(r \zeta)\right), \varphi(z)\right) d \sigma_{n}(\zeta)<\infty .
$$

Let $f \in \mathcal{B}\left(B_{m}\right)$. Then

$$
\left|f\left(\varphi\left(\phi_{z}(r \zeta)\right)\right)-f(\varphi(z))\right| \leq C \beta_{m}\left(\varphi\left(\phi_{z}(r \zeta)\right), \varphi(z)\right)
$$

by (2.2). Hence,

$$
\begin{aligned}
& \sup _{z \in B_{n}} \sup _{0<r<1} \int_{\partial B_{n}}\left|f\left(\varphi\left(\phi_{z}(r \zeta)\right)\right)-f(\varphi(z))\right| d \sigma_{n}(\zeta) \\
& \quad \leq C \sup _{z \in B_{n}} \sup _{0<r<1} \int_{\partial B_{n}} \beta_{m}\left(\varphi\left(\phi_{z}(r \zeta)\right), \varphi(z)\right) d \sigma_{n}(\zeta)<\infty .
\end{aligned}
$$

Using (2.5) with $p=1$, we have $f \circ \varphi \in \operatorname{BMOA}\left(B_{n}\right)$. So, (1.2) holds by the closed graph theorem. The proof of Theorem 1.1 is complete.

4.2. A necessary condition. The space $\operatorname{BMOA}\left(B_{n}\right)$ is not a lattice, so it is not expected that (1.2) is equivalent to a restriction on $\left|\varphi^{*}(\zeta)\right|, \zeta \in \partial B_{n}$. However, applying Lemma 3.2, we obtain a related explicit condition, which is necessary for (1.2).

Proposition 4.1. Let $\varphi: B_{n} \rightarrow B_{m}$ be a holomorphic map. Assume that $C_{\varphi}$ : $\mathcal{B}\left(B_{m}\right) \rightarrow \operatorname{BMOA}\left(B_{n}\right)$ is a bounded operator. Then there exist constants $\varepsilon=$ $\varepsilon\left(n, m,\left\|C_{\varphi}\right\|_{\mathcal{B} \rightarrow \mathrm{BMOA}}\right)>0$ and $C=C(n)>0$ such that

$$
\int_{\partial B_{n}} \exp \left(\varepsilon \log \frac{1}{1-\left|\varphi^{*}(\zeta)\right|^{2}}\right)^{\frac{1}{2}} d \sigma_{n}(\zeta) \leq C .
$$

Proof. The operator $C_{\varphi}$ maps $\mathcal{B}\left(B_{m}\right)$ to the Hardy space $H^{2}\left(B_{n}\right)$. So, arguing as in the proof of the implication $(1.2) \Rightarrow(1.3)$, we obtain

$$
\sup _{0<r<1} \int_{\partial B_{n}} \log \frac{1}{1-|\varphi(r \zeta)|^{2}} d \sigma_{n}(\zeta)<\infty
$$

Hence,

$$
\int_{\partial B_{n}} \log \frac{1}{1-\left|\varphi^{*}(\zeta)\right|^{2}} d \sigma_{n}(\zeta)<\infty
$$

by Fatou's lemma. In particular,

$$
\left|\varphi^{*}(\zeta)\right|<1
$$

for $\sigma_{n}$-almost every $\zeta \in \partial B_{n}$. If (4.2) holds, then

$$
f\left(\varphi^{*}(\zeta)\right)=\lim _{r \rightarrow 1-} f(\varphi(r \zeta))=(f \circ \varphi)^{*}(\zeta)
$$

for any $f \in H\left(B_{m}\right)$. 
Now, let the constant $\tau=\tau_{m, 1}>0$ and the functions $F_{j, x} \in \mathcal{B}\left(B_{m}\right), 1 \leq$ $j \leq J, 0 \leq x \leq 1$, be those provided by Lemma 3.2 with $p=1$. We have $\left\|F_{j, x} \circ \varphi\right\|_{\mathrm{BMOA}\left(B_{n}\right)} \leq\left\|C_{\varphi}\right\|_{\mathcal{B} \rightarrow \mathrm{BMOA}}$. Thus, for $\delta=A(n)\left\|C_{\varphi}\right\|_{\mathcal{B} \rightarrow \mathrm{BMOA}}^{-1}$, (4.3) and (2.4) guarantee that

$$
\begin{aligned}
\int_{\partial B_{n}} \exp \left(\delta\left|F_{j, x}\left(\varphi^{*}(\zeta)\right)\right|\right) d \sigma_{n}(\zeta) & =\int_{\partial B_{n}} \exp \left(\delta\left|\left(F_{j, x} \circ \varphi\right)^{*}(\zeta)\right|\right) d \sigma_{n}(\zeta) \\
& \leq C(n), \quad 1 \leq j \leq J, 0 \leq x \leq 1
\end{aligned}
$$

where the constants $A(n)>0$ and $C(n)>0$ are those provided by the JohnNirenberg theorem for $\operatorname{BMOA}\left(B_{n}\right)$. Therefore,

$$
\begin{aligned}
C(n) & \geq \int_{\partial B_{n}} \frac{1}{J} \sum_{j=1}^{J} \int_{0}^{1} \exp \left(\delta\left|F_{j, x}\left(\varphi^{*}(\zeta)\right)\right|\right) d x d \sigma_{n}(\zeta) \\
& \geq \int_{\partial B_{n}} \exp \left(\frac{\delta}{J} \sum_{j=1}^{J} \int_{0}^{1}\left|F_{j, x}\left(\varphi^{*}(\zeta)\right)\right| d x\right) d \sigma_{n}(\zeta) \\
& \geq \int_{\partial B_{n}} \exp \left(\frac{\tau \delta}{J} \sqrt{\log \frac{1}{1-\left|\varphi^{*}(\zeta)\right|^{2}}}\right) d \sigma_{n}(\zeta)
\end{aligned}
$$

by Fubini's theorem, Jensen's inequality and Lemma 3.2

\section{REFERENCES}

1. A. B. Aleksandrov, Function theory in the ball, Current problems in mathematics. Fundamental directions, Vol. 8, Itogi Nauki i Tekhniki, Akad. Nauk SSSR Vsesoyuz. Inst. Nauchn. i Tekhn. Inform., Moscow, 1985, pp. 115-190, 274 (Russian); English transl., Encyclopaedia Math. Sci., vol. 8, Springer-Verlag, Berlin, 1994, pp. 107-178. MR850487 (88b:32002)

2. A. B. Aleksandrov, Proper holomorphic mappings from the ball to the polydisk, Dokl. Akad. Nauk SSSR 286 (1986), no. 1, 11-15 (Russian); English transl.: Soviet Math. Dokl. 33 (1986), no. 1, 1-5. MR822088 (87g:32029)

3. O. Blasco, M. Lindström, and J. Taskinen, Bloch-to-BMOA compositions in several complex variables, Complex Var. Theory Appl. 50 (2005), no. 14, 1061-1080. MR2175841 (2006f:47027)

4. B. R. Choe, W. Ramey, and D. Ullrich, Bloch-to-BMOA pullbacks on the disk, Proc. Amer. Math. Soc. 125 (1997), no. 10, 2987-2996. MR.1396971 (97m:47039)

5. C. C. Cowen and B. D. MacCluer, Composition operators on spaces of analytic functions, Studies in Advanced Mathematics, CRC Press, Boca Raton, FL, 1995. MR1397026 (97i:47056)

6. J. B. Garnett, Bounded analytic functions, Pure and Applied Mathematics, vol. 96, Academic Press Inc., New York, 1981. MR628971 (83g:30037)

7. E. G. Kwon, On analytic functions of Bergman BMO in the ball, Canad. Math. Bull. 42 (1999), no. 1, 97-103. MR1695858 (2001a:32005)

8. E. G. Kwon, Hyperbolic g-function and Bloch pullback operators, J. Math. Anal. Appl. 309 (2005), no. 2, 626-637. MR2154140 (2006d:30051)

9. S. Makhmutov and M. Tjani, Composition operators on some Möbius invariant Banach spaces, Bull. Austral. Math. Soc. 62 (2000), no. 1, 1-19. MR.1775882 (2001i:47041)

10. W. Ramey and D. Ullrich, Bounded mean oscillation of Bloch pull-backs, Math. Ann. 291 (1991), no. 4, 591-606. MR1135533 (92i:32004)

11. J. Ryll and P. Wojtaszczyk, On homogeneous polynomials on a complex ball, Trans. Amer. Math. Soc. 276 (1983), no. 1, 107-116. MR684495 (84f:32004)

12. J. H. Shapiro, Composition operators and classical function theory, Universitext: Tracts in Mathematics, Springer-Verlag, New York, 1993. MR1237406 (94k:47049) 
13. W. Smith and R. Zhao, Composition operators mapping into the $Q_{p}$ spaces, Analysis $\mathbf{1 7}$ (1997), no. 2-3, 239-263. MR 1486367 (98j:47075)

14. S. Yamashita, Holomorphic functions of hyperbolically bounded mean oscillation, Boll. Un. Mat. Ital. B (6) 5 (1986), no. 3, 983-1000. MR871709 (88e:30092)

15. K. Zhu, Spaces of holomorphic functions in the unit ball, Graduate Texts in Mathematics, vol. 226, Springer-Verlag, New York, 2005. MR2115155 (2006d:46035)

16. A. Zygmund, Trigonometric series. 2nd ed. Vols. I, II, Cambridge University Press, New York, 1959. MR0107776 (21:6498)

St. Petersburg Branch of V. A. Steklov Mathematical Institute, Fontanka 27, St. Petersburg 191023, Russia

E-mail address: dubtsov@pdmi.ras.ru 